\title{
Diagnóstico de nematodos gastrointestinales resistentes a bencimidazoles e imidazotiazoles en un rebaño caprino de Yucatán, México.
}

\section{Artículo Original}

Juan F. Torres-Acostaํㅜㄹ Marbel S. Villarroel-Álvarez ${ }^{1}$, Flavio Rodríguez-Arévalo ${ }^{1}$, Isidro Gutiérrez-Segura ${ }^{2}$, Miguel A. Alonso-Díaz 3 .

${ }^{1}$ Facultad de Medicina Veterinaria y Zootecnia, Universidad Autónoma de Yucatán, Mérida, Yucatán. ${ }^{2}$ Escuela de Medicina Veterinaria y Zootecnia, Universidad Autónoma de Guerrero. ${ }^{3}$ Centro de Enseñanza, Investigación y Extensión en Ganadería Tropical, Universidad Nacional Autónoma de México, México, D.F., México.

\section{RESUMEN.}

Introducción. El uso de antihelmínticos $(\mathrm{AH})$ para el control de nematodos gastrointestinales (NGI) en caprinos se ve amenazado por el surgimiento de cepas de NGI resistentes a AH. No existe información de la presencia de NGI resistentes en rebaños caprinos de México y Yucatán. El objetivo fue determinar la presencia de NGI resistentes a bencimidazoles (BZ), imidazotiazoles (LEV) y lactonas macrocíclicas (LM) en un rebaño caprino de Yucatán, México, mediante la prueba de reducción de cuentas de huevos fecales. Material y métodos. La presencia de nematodos resistentes a BZ, LEV y LM fue determinada mediante la prueba de reducción de cuentas de huevos fecales. La técnica de conteo de huevos fecales de McMaster modificada se utilizó para seleccionar sesenta cabras adultas con más de 150 huevos por gramo de heces (HPG). Estos animales fueron distribuidos al azar en cuatro grupos de 14 ó 15 animales. El grupo 1 permaneció sin tratamiento $\mathrm{AH}(\mathrm{T})$. El grupo 2 fue tratado con BZ $(7.6 \mathrm{mg} / \mathrm{kg}$ de peso vivo (PV) de albendazol per os). El grupo 3 con LEV (12mg/kg de PV de levamisol por vía subcutánea) y el grupo 4 con LM $(0.2 \mathrm{mg} / \mathrm{kg}$ de PV de moxidectina por vía subcutánea). Los animales fueron pesados individualmente y las drogas fueron administradas de acuerdo al peso. Los animales fueron aislados de alimento durante 16 horas antes del tratamiento $\mathrm{AH}$. Todos los animales fueron muestreados nuevamente el día 12 post-tratamiento. Los resultados de HPG del segundo muestreo se usaron para determinar el porcentaje de reducción de HPG (\%R) y el intervalo de confianza 95\% (IC95\%). Se realizó un cultivo fecal por cada grupo estudiado para identificar los géneros de larvas en una muestra de cincuenta larvas infectantes de cada grupo.

Resultados. No se encontró resistencia contra LM en el rebaño estudiado. Se encontraron NGI resistentes a BZ $(\% \mathrm{R}=89$, IC95\%=77-94) y LEV $(\% \mathrm{R}=82$, IC95\%=59-92). Haemonchus spp. fue el

Solicitud de sobretiros: Dr. J.F.J. Torres-Acosta, Fac. de Medicina Veterinaria y Zootecnia, Universidad Autónoma de Yucatán,Km. 15.5 carretera Mérida-Xmatkuil, Mérida, Yucatán, México. E-mail: tacosta@tunku.uady.mx Recibido el 14/Febrero/2002. Aceptado para publicación el 27/Noviembre/2002. 
JFJ Torres-Acosta, MS Villarroel-Álvarez, FJ Rodríguez-Arévalo y col.

género de NGI resistente a BZ. Los géneros resistentes a LEV fueron Trichostrongylus spp. (96\%) y Oesophagostomun spp. (4\%).

Discusión. La presencia de NGI resistentes a LEV y BZ pudo ser causada por varios años de uso de dosis sub-terapéuticas de ambos AH en el rebaño. Estas dosis sub-terapéuticas fueron obtenidas de la etiqueta de las drogas utilizadas. La falta de rotación anual de drogas en el rebaño pudo haber contribuido al desarrollo de resistencia.

(Rev Biomed 2003; 14:75-81)

Palabras clave: Resistencia antihelmíntica, nematodos gastrointestinales, caprinos, México.

\section{SUMMARY.}

Diagnosis of gastrointestinal nematodes resistant to bencimidazols and imidazothiazols in a goat herd in Yucatan, Mexico.

Introduction. The use of anthelmintics $(\mathrm{AH})$ for the control of gastrointestinal nematodes (GIN) in goats is threatened by the emergence of resistant strains of GIN. No information is available on the presence of resistant nematodes in goat herds of Mexico and Yucatan. The objective was to determine the presence of GIN resistant to bencimidazols (BZ), imidazothiazols (LEV), and macrocyclic lactones (ML) in a goat herd of Yucatan, Mexico by means of the faecal egg count reduction test.

Materials and methods. Presence of GIN resistant to BZ, LEV and ML was determined through the Faecal Egg Count Reduction Test. A modified McMaster technique was used to select sixty adult goats with faecal egg counts above 150 eggs per gram of faeces (EPG). These animals were randomly distributed in four groups of 14 to 15 animals. Group 1 remained without AH treatment (T). Group 2 was treated with BZ $(7.6 \mathrm{mg} / \mathrm{kg}$ liveweight of albendazol per os). Group 3 with LEV ( $12 \mathrm{mg} / \mathrm{kg}$ liveweight of levamisol subcutaneously) and group 4 with ML $((0.2 \mathrm{mg} / \mathrm{kg}$ liveweight of moxidectin, subcutaneously). Animals were individually weighed and drugs administered according to weight. Animals were refrained of feed during 16 hours before $\mathrm{AH}$ treatment. All the animals were sampled again on day 12 post-treatment. Results of the second sampling were used to determine percentage reduction of EPG $(\% \mathrm{R})$ and the $95 \%$ confidence interval (IC95\%). A bulk faecal culture was made for each treatment group to identify the genus of larvae in a sample of fifty infective larvae in each group.

Results. No resistance against ML was found in the herd. There were GIN resistant to BZ (\% R=89, IC95\% $=77-94)$ and LEV (\% R=82, IC95\%=59-92). The genus of GIN resistant to BZ was Haemonchus spp. The genera of GIN resistant to LEV were Trichostrongylus spp. (96\%) and Oesophagostomun spp. (4\%).

Discusion. Presence of GIN resistant to LEV and BZ may have been caused by several years of subtherapeutic dosing of both AH in the herd. These subtherapeutic doses were obtained from the label of the drugs used. The lack of yearly drug rotation in the herd may have contributed towards the development of resistance. (Rev Biomed 2003; 14:75-81)

Key words: Anthelmintic resistance, gastrointestinal nematodes, goats, México.

\section{INTRODUCCIÓN.}

Los nematodos gastrointestinales (NGI) disminuyen la producción de los caprinos en los sistemas de producción que utilizan pastoreo y ramoneo causando retraso de crecimiento, baja producción de leche e incluso la muerte (1). La herramienta más utilizada para el control de NGI es el tratamiento antihelmíntico (AH). Sin embargo, el uso indiscriminado y muchas veces inadecuado de los AH ha permitido el desarrollo de resistencia en las poblaciones de NGI. La resistencia a los antihelmínticos (RA) es la presencia de una gran proporción de NGI capaces de tolerar y sobrevivir a la aplicación de dosis normales de una o más familias de desparasitantes (2). La RA se hereda a generaciones futuras de NGI, lo que significa que una vez que se tienen NGI resistentes en una explotación éstos estarán presentes indefinidamente (3). La existencia de NGI resistentes amenaza la sustentabilidad de los métodos de control de NGI

\section{Revista Biomédica}




\section{Nematodos resistentes a antihelmínticos en caprinos.}

dependientes del uso de esas drogas. El enorme costo del desarrollo y registro de nuevos AH hace cada vez más difícil para las empresas farmacéuticas lanzar principios activos distintos a los de las tres familias de AH existentes (bencimidazoles, imidazotiazoles y lactonas macrocíclicas). Esto hace más imperiosa la necesidad de conservar la eficacia de las drogas existentes y de cualquier nuevo producto para el futuro. La RA es un problema que se ha detectado en todo el mundo. En América Latina, particularmente en Sudamérica, existe gran cantidad de rebaños ovinos que tienen NGI resistentes (4). En México ha sido insuficiente el esfuerzo de diagnóstico que se ha realizado. La falta de diagnóstico no significa ausencia del problema, sino por el contrario expresa carencias que van desde pasar por alto la problemática en el campo, hasta la imposibilidad de realizar el diagnóstico de laboratorio o el desconocimiento de las pruebas utilizadas para el diagnóstico (5). Es necesario generar información referente a la RA para conocer el nivel de eficacia de las drogas, así como identificar los géneros de NGI resistentes, con la finalidad de desarrollar e implementar mecanismos de control integral encaminados a optimizar la eficacia de los productos químicos y contrarrestar el impacto económico ocasionado por los NGI en los sistemas de producción caprina. El objetivo del presente trabajo fue determinar la presencia de NGI resistentes a bencimidazoles (BZ), imidazotiazoles y lactonas macrocíclicas (LM) en un rebaño caprino de Yucatán, México.

\section{MATERIAL Y MÉTODOS.}

Localización. El presente trabajo se realizó durante los meses de abril a mayo de 2001 en un rebaño localizado en el municipio de Mérida, Yucatán, México (2050' N y 8940' W) (6).

Animales. Se utilizaron 84 cabras adultas criollas, de las cuales se seleccionaron 60 de acuerdo a las recomendaciones de Coles y col. (7): a) eliminación de al menos 150 huevos por gramo de heces (hpg) (determinado mediante la técnica de McMaster modificada) (8) y b) no haber sido desparasitadas con $\mathrm{AH}$ durante las últimas 8-12 semanas. Los animales estuvieron bajo un esquema de pastoreo semi-extensivo donde tenían acceso al agostadero nativo siete horas/día. Posteriormente se confinaban para recibir $100 \mathrm{~g}$ de alimento concentrado con $16 \%$ de proteína cruda y agua ad libitum.

Historial de desparasitación del rebaño. El rebaño de estudio tuvo un historial de uso continuo de BZ desde 1985, en que el rebaño fue adquirido, hasta 1992. Durante ese período se utilizaron los BZ febendazole y febantel tal como se indicó en la etiqueta para ovinos y caprinos $(5 \mathrm{mg} / \mathrm{kg})$. El BZ fue utilizado en varias formas a lo largo del historial de uso. Inicialmente, cuando el rebaño era pequeño (máximo 50 animales), se desparasitó con BZ a todos los animales únicamente cuando aparecían signos clínicos en algún(os) animal(es). Esto significó un uso esporádico con períodos de varios meses sin tratamiento (entre 1985 y 1987). Posteriormente, cuando el rebaño ya incluía cerca de 150 animales en pastoreo/ramoneo, se comenzó a usar un esquema de tres a cuatro desparasitaciones en la época de lluvia y una en época de seca (desde el verano de 1988 hasta el verano de 1992). Posteriormente (19931997) se utilizó levamisol (LEV) en un esquema de dos tratamientos a todos los animales en época de lluvia y uno al inicio de la época de seca. La dosis usada fue la recomendada en la etiqueta para ovinos y caprinos $(7.5 \mathrm{mg} / \mathrm{kg})$. A partir de 1997 y hasta el 2000 se usó LEV en forma selectiva, es decir, solamente en aquellos animales con signos de emaciación y diarrea (preferentemente con diagnóstico de MacMaster confirmatorio). El año 2001 se empezó a hacer la rotación anual de $\mathrm{AH}$ (BZ y LEV) pero usando el esquema selectivo de tratamiento indicado anteriormente. La LM moxidectina comenzó a ser utilizada en un grupo de 16 cabritos desde 1997. Esta LM es usada cada 28 días en el grupo experimental mencionado a una dosis de $0.2 \mathrm{mg} / \mathrm{kg}$ por periodos de 6 a 10 meses de cada año.

Diseño experimental. Se utilizó un diseño en el que se distribuyeron las unidades experimentales en cuatro grupos de 14 ó 15 animales cada uno. El grupo 1 permaneció como testigo (sin tratamiento $\mathrm{AH}$ ) (T). El grupo 2 fue tratado con BZ $(7.6 \mathrm{mg} / \mathrm{kg}$ de peso 
JFJ Torres-Acosta, MS Villarroel-Álvarez, FJ Rodríguez-Arévalo y col.

vivo de albendazol per os). El grupo 3 con LEV $(12 \mathrm{mg} / \mathrm{kg}$ de peso vivo (PV) de levamisol por vía subcutánea) y el grupo 4 con una $\mathrm{LM}(0.2 \mathrm{mg} / \mathrm{kg}$ de PV de moxidectina por vía subcutánea). Las dosis de AH utilizadas son las recomendadas para caprinos por Hoste (9). Los animales de cada grupo fueron pesados individualmente para ser desparasitados de acuerdo a su peso. Se mantuvieron sin alimento durante 16 horas previas a la desparasitación. El día de la desparasitación fue considerado como día 0. Todos los animales fueron muestreados nuevamente el día 12 post-tratamiento para realizar un segundo McMaster (7).

Prueba de reducción de huevos en heces. Para determinar el grado de resistencia antihelmíntica en los nematodos, se utilizó el porcentaje de reducción de HPG (huevos por gramo de heces) (\%R) y el intervalo de confianza 95\% (IC95\%)(7). Para esto se utilizó el programa de computo RESO.EXE.

Cultivo de heces, cosecha de larvas infectantes e identificación de larvas de NGI. Las heces obtenidas de los animales en el día 0 del estudio sirvieron para elaborar 4 cultivos de heces, uno por cada grupo estudiado de acuerdo a la técnica descrita por Rodríguez y col. (10). El cultivo de heces se mantuvo a temperatura ambiente durante cinco días. Se recuperaron las larvas infectantes de NGI con la técnica de Corticelli-Lai (10). Los géneros de las larvas infectantes del orden strongylida fueron determinados mediante la identificación de 50 larvas infectantes usando como criterios la morfología descrita en MAFF (11) y las medidas descritas por Bowman y Lynn (12). El día 12 postratamiento se elaboró un cultivo por cada grupo experimental y se procedió de la manera ya descrita hasta identificar los géneros presentes en cada cultivo.

Análisis estadístico. Se utilizó el programa de computo RESO.EXE (13) para calcular la media aritmética de cada grupo, el \%R de HPG y el IC95\%. Se consideraron resistentes los grupos que promediaron un \% Renor a 95\% e IC95\% inferior a $90 \%$ (7).

\section{Revista Biomédica}

\section{RESULTADOS.}

En el cuadro 1, se muestran los resultados del efecto del tratamiento AH sobre el porcentaje de reducción de HPG de nematodos gastrointestinales. Se observa que el mayor porcentaje de reducción se obtuvo en el grupo tratado con LM (\%R=100). Se encontraron NGI resistentes a BZ $(\% \mathrm{R}=89$, IC95\%=77-94) y a LEV $(\% \mathrm{R}=82$, IC95\%=59-92).

La identificación de larvas infectantes de los cultivos de heces de cada grupo mostraron que el género de NGI resistentes a BZ fue Haemonchus spp. (100\%). Por otro lado, los géneros de NGI resistentes a LEV fueron Trichostrongylus spp. (96\%) y Oesophagostomum spp. (4\%). En el grupo control se encontraron larvas de Haemonchus spp. (30\%), Trichostrongylus spp. (64\%) y Oesophagostomum spp. (6\%). No hubo crecimiento larvario en el grupo tratado con LM.

\section{DISCUSIÓN.}

El presente estudio es el primer reporte de NGI resistentes a LEV y BZ en caprinos de México. Únicamente la LM, moxidectina, tuvo una eficacia de $100 \%$ contra los géneros de NGI encontrados en el rebaño estudiado. Esto confirma la ausencia de resistencia contra la familia de las LM en los NGI del rebaño. Esto pudo deberse a que en este rebaño las LM se han utilizado solamente en pequeños grupos experimentales y por períodos cortos desde 1997. Esta alta eficacia confirma los resultados de estudios previos en caprinos, tanto de farmacocinética (14) como de persistencia de la eficacia (15). A pesar de esta alta eficacia, la moxidectina no está autorizada para su uso en el control de NGI de caprinos a nivel mundial (16). Por otro lado, el presente estudio mostró la presencia de cepas de NGI resistentes contra LEV y BZ. La resistencia encontrada contra levamisol confirma la presencia de NGI resistentes contra AH de la familia de los LEV y la resistencia encontrada contra albendazol confirma la presencia de NGI resistentes contra los AH de la familia de los BZ (incluyendo los pro-bencimidazoles) (7). La presencia de NGI resistentes a los BZ ha sido más reportada que aquella contra los LEV (2). Esto tal vez se debe a 


\section{Nematodos resistentes a antihelmínticos en caprinos.}

\section{Cuadro 1}

Eficacia de los tratamientos antihelmínticos albendazol, levamisol y moxidectina sobre el porcentaje de reducción de huevos de nematodos gastrointestinales en un rebaño caprino del estado de Yucatán, México.

\begin{tabular}{|c|c|c|c|c|c|c|}
\hline \multirow[t]{2}{*}{ Tratamiento } & \multirow{2}{*}{$\begin{array}{c}\text { Dosis } \\
\text { (vía de } \\
\text { administración) }\end{array}$} & \multirow{2}{*}{$\mathrm{N}$} & \multicolumn{2}{|c|}{ Media hpga } & \multirow{2}{*}{$\begin{array}{l}\text { Porcentaje de } \\
\text { Reducción }^{\mathrm{b}}\end{array}$} & \multirow{2}{*}{$\begin{array}{c}\text { Intervalo de } \\
\text { Confianza 95\% }\end{array}$} \\
\hline & & & Pre-tratamiento & Post-tratamiento & & \\
\hline Control & & 15 & 450 & 546.7 & - & - \\
\hline Albendazol & $7.6 \mathrm{mg} / \mathrm{kg}$, (oral) & 15 & 430 & 66.67 & 89 & $77-94$ \\
\hline Levamisol & 12mg/kg, (subcutánea) & 14 & 517 & 107.1 & 82 & $59-92$ \\
\hline Moxidectina & 0.2mg/kg, (subcutánea) & 15 & 423 & 0 & 100 & - \\
\hline
\end{tabular}

${ }^{\text {ahpg }}=$ huevos por gramo de heces

${ }^{b}$ Porcentaje de reducción de huevos calculado por el programa RESO.EXE

'Intervalo de confianza 95\% calculado por el programa RESO.EXE

que en muchos trabajos de diagnóstico de NGI resistentes sólo se incluye la resistencia contra BZ debido a las restricciones legales del uso de LEV en esta especie (17). Sin embargo, existen casos donde la prevalencia de NGI resistentes contra LEV es menor que aquella contra BZ (18). Hay dos posibles razones de la presencia de NGI resistentes contra estas dos familias de AH: a) uso inadecuado de dosis por largos períodos de tiempo (varios años) y b) ausencia de rotación de familias de $\mathrm{AH}$. En lo que respecta al uso inadecuado de dosis, éste ocurrió por muchos años en el rebaño de estudio ya que se utilizaron dosis recomendadas por los fabricantes de cada $\mathrm{AH}$. Ahora se sabe que las dosis recomendadas por estos fabricantes son inferiores que las requeridas por caprinos para alcanzar una eficacia óptima (dosis subterapéuticas). Hoste (9) ha sugerido dosis adecuadas para los caprinos basando sus recomendaciones en los escasos estudios de farmacodinamia de antihelmínticos en caprinos (19-22). Por lo tanto, los BZ fueron usados en dosis sub-terapéuticas $(5 \mathrm{mg} / \mathrm{kg})$, recomendadas por el fabricante, por un período de 7 años. El LEV fue utilizado en dosis subterapéutica $(7.5 \mathrm{mg} / \mathrm{kg}$ ) por 7 años consecutivos con un promedio de tres aplicaciones por año. La aplicación de dosis subterapéuticas, tanto de LEV como de BZ en caprinos, es un fenómeno común. Aún en trabajos de prevalencia de NGI resistentes a AH se han utilizado dosis más bajas que las recomendadas $(17,18)$. En muchos casos, la resistencia reportada puede estar confundida con baja eficacia debido a que no se alcanzó la dosis terapéutica adecuada. Sin embargo, éste no fue el caso en el presente estudio ya que se utilizaron las dosis y vías de aplicación recomendadas por Hoste (9).

La ausencia prolongada de una rotación anual de las diferentes familias de $\mathrm{AH}$ de amplio espectro pudo contribuir a la creación de NGI resistentes (5, 23). El uso constante durante años de una sola familia de AH contribuye a seleccionar las cepas resistentes de NGI. Esta rotación parece ser especialmente importante en drogas sin larga persistencia como BZ y LEV (5). Es de llamar la atención que, a pesar de haberse usado varios años las drogas AH en forma sub-terapéutica, en este rebaño la eficacia no es excesivamente baja como las que se reportan en rebaños ovinos de países de Sudamérica $(4,5)$. Esto puede deberse a que las drogas fueron usadas en pocas ocasiones al año. En el caso de la resistencia contra los BZ orales, la relativamente baja eficacia puede ser mejorada mediante estrategias de dosificación encaminadas a mejorar su biodisponibilidad y, por ende, la eficacia (24). Estas consisten en dietar a los animales $24 \mathrm{~h}$ antes del tratamiento $(5,24)$ y aplicar la dosis dividida en intervalos de $12 \mathrm{~h}(24,25)$.

El presente estudio muestra un complicado escenario de géneros de NGI resistentes a diferentes familias de AH. Haemonchus spp. fue resistente a BZ, en tanto que Trichostrongylus spp. y Oesophagostomum spp. fueron resistentes a LEV. 


\section{JFJ Torres-Acosta, MS Villarroel-Álvarez, FJ Rodríguez-Arévalo y col.}

La tendencia de Haemonchus spp. a ser resistente a los AH de la familia de los BZ ha sido reportada con anterioridad (18). Los parásitos resistentes a los BZ se caracterizan por la pérdida de afinidad de los sitios de unión de estas drogas en los microtúbulos del parásito. Mediante la técnica de reacción en cadena de la polimerasa se ha encontrado el reemplazamiento del aminoácido fenilalanina por tirosina (mutación) en la posición 200 de un gen Btubulina de aislamientos de Haemonchus spp. resistentes a BZ (26). Las modificaciones genéticas ocurren rápidamente en las poblaciones de Haemonchus spp. expuestos a bencimidazoles debido a su alto potencial biótico (elevada capacidad de producción de huevos por las hembras). La producción de grandes cantidades de huevos de los NGI resistentes implica una rápida dilución genética en la población que conforma el refugio, acelerando la aparición de poblaciones resistentes (2). Algunos investigadores sugieren que la resistencia de Trichsotrongylus spp. contra el LEV puede ser debida a alteraciones a nivel de receptor (26). No obstante, no se encontró evidencia previa de la selectividad de la resistencia de Trichostrongylus spp. y Oesophagostomum spp. por LEV como la reportada en este estudio.

En conclusión, en el rebaño caprino estudiado existen NGI resistentes a BZ y LEV. Los NGI del rebaño estudiado son susceptibles a las LM. Se encontró que Haemonchus spp. fue resistente a BZ, mientras que Trichostrongylus spp. y Oesophagostomum spp. fueron resistentes a LEV.

\section{AGRADECIMIENTOS.}

Agradecemos al MC Alfredo Cuellar Ordaz, académico de la Facultad de Estudios Superiores de Cuautitlán, UNAM, por proveernos el programa australiano RESO.EXE.

\section{REFERENCIAS.}

1.- Smith MC, Sherman DM. Goat Medicine. 1st Ed. Malvern, Pennsylvania. Lea and Febiger. 1994.

2.- Jackson F. Anthelmintic resistance in goats. 1er. Curso Internacional: Nuevas perspectivas en el diagnostico de nematodos gastrointestinales en pequeños rumiantes. Mérida, Yucatán, México. Universidad Autónoma de Yucatán.
2000; p. 38-48.

3.- Nari A, Hansen J. Resistance of ecto and endo-parasites: current and future solutions, 67th General Session. International Committee. OIE. Paris; 17 - 21 Mayo, 1999.

4.- Waller PJ. Anthelmintic resistance. Vet Parasitol 1997; 72: 391-412.

5.- Nari A. Diagnóstico y control de resistencia antihelmíntica en pequeños rumiantes. Memoria electrónica del 2 do. Congreso Latinoamericano de Pequeños Rumiantes y Camélidos Americanos. Mérida, Yucatán, México. 2001.

6.- Bassol DA. Geografía Económica de México. $3^{\mathrm{a}}$ ed. México: Editorial Trillas. 1975.

7.- Coles GC, Bauer C, Borgsteede FHM, Greerts S, Taylor MA, Waller, PJ. Methods for the detection of anthelmintic resistance in nematodes of veteriany importance. Vet Parasitol 1992; 44: 35-44.

8.- Hansen J, Perry B. The epidemiology, diagnosis and control of helminth parasites of ruminants. $1^{\text {st }}$ ed. Nairobi, Kenya. FAO-ILRAD, 1994.

9.- Hoste H. Control of gastrointestinal nematodes through anthelmintics in dairy goats. 1er. Curso Internacional: Nuevas perspectivas en el diagnóstico y control de nematodos gastrointestinales en pequeños rumiantes. Mérida, Yucatán, México. Universidad Autónoma de Yucatán. 2000; pp. 3237.

10.- Rodríguez VRI, Domínguez AJL, Cob, JLA. Técnicas diagnósticas de parasitología veterinaria. Mérida, Yucatán. México: Universidad Autónoma de Yucatán. 1994.

11.- Ministry of Agriculture, Fisheries, and Food. Helminthology. En: Manual of Veterinary Parasitological Laboratory Techniques. Reference book 418. $3^{\text {rd }}$ ed. London, England. Ministry of Agriculture, Fisheries and Food. Her Majesty's Stationary Office. 1986. p. 1-65.

12.- Bowman DD, Lynn RC. Georgis' Parasitology for Veterinarians. $7^{\text {th }}$ ed. Philadelphia: W.B. Saunders Co.; 1999. p. 320-5.

13.- Anonymous. Report of the Working Party for the Animal Health Committee of the Staning Committee on Agriculture. SCA Tech. Rep. Ser. No. 28, Anthelmintic resitance, CSIRO, Australia; 1989.

14.- Escudero E, Carceles CM, Diaz MS, Sutra JF, Galtier P,

\section{Revista Biomédica}




\section{Nematodos resistentes a antihelmínticos en caprinos.}

Alvinaire M. Pharmacokinetics of moxidectin and doramectin in goats. Res Vet Sci 1999; 67: 177-81.

15.- Torres-Acosta JFJ, Jacobs DE. Duration of activity of oral moxidectin against Haemonchus contortus, Teladorsagia circumcincta, and Trichostrongylus colubriformis in goats. Vet Rec 1999; 144: 648-9.

16.- Cobb RM, Murphy AW. Use of moxidectin in goats in New Zealand. Vet Rec 1995; 137: 496.

17.- Chartier C, Pors I, Hibert J, Rocheteau D, Benoit C, Bernard N. Prevalence of anthelmintic resistant nematodes in sheep and goats in Western France. Small Rum Res 1998; 29: 33- 41.

18.- Dorny P, Claerebout E, Vercruysse J, Sani R, Jalila A. Anthelmintic resistance in goats in peninsular Malaysia. Vet Parasitol 1994; 55: 327-42.

19. Galtier P, Escoula R, Camguilhem R, Alvinaire M. Comparative bioavailability of levamisole in non lactating ewes and goats. Ann Réch Vét 1981; 12: 109-15.

20. Bogan J, Benoit E, Delatour P. Pharmacokinetics of oxfendazole in goats: a comparison with sheep. J Vet Pharm 1987; 10: 305-9

21.- Coles GC, Giordano-Fenton DJ, Tritschler JPLl. Efficacy of levamisole against inmature and mature nematodes in goats with induced infections. Am J Vet Res 1989; 50: 1074-5.

22.- Sangster NC, Rickard J.M., Hennessy D.R., Steel J.W. Disposition of oxfendazole in goats and efficacy compared with sheep. Res Vet Sci 1991; 51: 258-63.

23.- Coles GC, Roush RT. Slowing the spread of anthelmintic resistant nematodes of sheep and goats in the United Kingdom. Vet Rec 1992; 130: 505-10.

24.- Jackson F. Methods for extending the efficacy of anthelmintics. 1er. Curso Internacional: Nuevas perspectivas en el diagnóstico de nematodos gastrointestinales en pequeños rumiantes. Mérida, Yucatán, México. Universidad Autónoma de Yucatán; 2000. p. 49-52.

25.- Henessy DR. Modifying the formulation of delivery mechanism to increase the activity of anthelmintic compounds. Vet Parasitol 1997; 72: 367-90.

26.- Kohler P. The biochemical basis of anthelmintic action and resistance. Int J Parasitol 2001; 31: 336-45. 